Supporting Information

\title{
Total Synthesis of Diocollettines A via an Acid-Promoted Oxa-Michael-Aldol-Acetalization Cascade
}

\author{
Misaki Kawamoto, Shuntaro Sato, Masaru Enomoto, Yusuke Ogura, Shigefumi Kuwahara* \\ Laboratory of Applied Bioorganic Chemistry, Graduate School of Agricultural Science, Tohoku University \\ 468-1 Aramaki Aza-Aoba, Aoba-ku, Sendai 980-0845, Japan \\ shigefumi.kuwahara.e1@tohoku.ac.jp
}

\section{Table of Contents}

General information 


\section{General Information}

IR spectra were recorded by a Jasco FT/IR-4100 spectrometer using an ATR (ZnSe) attachment. NMR spectra were recorded with TMS as an internal standard in $\mathrm{CDCl}_{3}$ by a Varian 400 -MRTT spectrometer $\left(400 \mathrm{MHz}\right.$ for ${ }^{1} \mathrm{H}$ and $100 \mathrm{MHz}$ for ${ }^{13} \mathrm{C}$ ) or a Varian 600 -TT spectrometer $\left(600 \mathrm{MHz}\right.$ for ${ }^{1} \mathrm{H}$ and $150 \mathrm{MHz}$ for ${ }^{13} \mathrm{C}$ ) unless otherwise stated. Optical rotation values were measured with a Jasco P-2200 polarimeter. Mass spectra were obtained with JEOL JMS-700 double-focusing magnetic sector mass spectrometer operated in the EI or FAB mode. Melting points were determined with a Yanaco MP-J3 apparatus and are uncorrected. Kanto Chemical silica gel 60N (spherical neutral, $40-50 \mu \mathrm{m}$ or $63-210 \mu \mathrm{m}$ ) was used for column chromatography. Analytical thin-layer chromatography was performed using Merck silica gel $60 \mathrm{~F}_{254}$ plates $(0.25 \mathrm{~mm}$ thick). Solvents for reactions were distilled prior to use: THF from $\mathrm{Na}$ and benzophenone; $\mathrm{CH}_{2} \mathrm{Cl}_{2}$ and $\mathrm{DMF}$ from $\mathrm{CaH}_{2} ; \mathrm{MeOH}$ from $\mathrm{Mg}(\mathrm{OMe})_{2}$. All air- or moisture-sensitive reactions were conducted under a nitrogen atmosphere. 


\section{Experimental Procedures and Characterization Data}

\section{(Z)-5-Hydroxy-1,7-diphenylhept-2-en-1-one (13).}

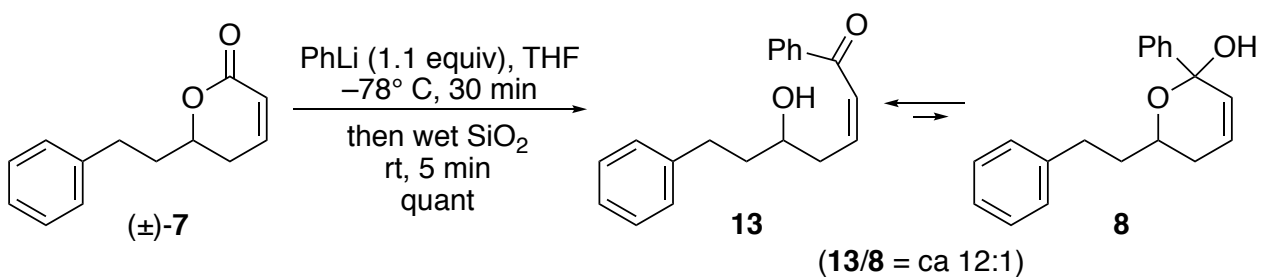

To a stirred solution of $( \pm)-7(31.3 \mathrm{mg}, 0.155 \mathrm{mmol})$ in THF (1.6 mL) was added dropwise a solution of PhLi (1.6 $\mathrm{M}$ in dibutyl ether, $0.110 \mathrm{~mL}, 0.171 \mathrm{mmol}$ ) at $-78^{\circ} \mathrm{C}$. After $30 \mathrm{~min}$ of stirring, silica gel was added at $-78^{\circ} \mathrm{C}$ and the resulting mixture was stirred at ambient temperature for $5 \mathrm{~min}$. The mixture was filtered through a pad of Celite and the filtrate was concentrated in vacuo to give an oil (44.2 mg, quant) composed of $\mathbf{1 3}$ and $\mathbf{8}$ in a ratio of 12:1 as analyzed by ${ }^{1} \mathrm{H}$ NMR. The oil was expeditiously purified by silica gel column chromatography (hexane/diethyl ether $=3: 1$ to $1: 1)$ to give a pure fraction consisting of $\mathbf{1 3}$ and $\mathbf{8}$ as a colorless oil (13/8=12:1) (see S6 and S7). IR: $v_{\max } 3459(\mathrm{~m}), 3028(\mathrm{w}), 1665(\mathrm{~m}), 1233(\mathrm{~s}) ;{ }^{1} \mathrm{H}$ NMR data for 13 (400 MHz, $\left.\mathrm{C}_{6} \mathrm{D}_{6}\right): \delta 1.56-$ $1.76(2 \mathrm{H}, \mathrm{m}), 2.52$ (1H, dddd, $J=1.2,4.0,7.6,14.2 \mathrm{~Hz}), 2.57-2.72(1 \mathrm{H}, \mathrm{m}, \mathrm{OH}), 2.65(1 \mathrm{H}, \mathrm{dd}, J=7.4,9.1 \mathrm{~Hz})$, $2.68(1 \mathrm{H}, \mathrm{dd}, J=7.2,9.1 \mathrm{~Hz}), 2.78(1 \mathrm{H}, \mathrm{ddd}, J=5.4,9.1,14.2 \mathrm{~Hz}), 3.56-3.66(1 \mathrm{H}, \mathrm{m}), 6.08(1 \mathrm{H}, \mathrm{dddd}, J=1.2$, 7.6, 9.111 .6$), 6.59(1 \mathrm{H}, \mathrm{td}, J=1.2,11.6 \mathrm{~Hz}), 7.00-7.21(8 \mathrm{H}, \mathrm{m}), 7.80-7.85(2 \mathrm{H}, \mathrm{m}) ;{ }^{13} \mathrm{C}$ NMR data for 13 [150 $\mathrm{MHz}, \mathrm{CD}_{3} \mathrm{CN}, \mathrm{CD}_{3} \mathrm{CN}$ as an internal standard $\left.(\delta 1.32 \mathrm{ppm})\right]$ : $\delta 32.6,38.2,40.1,70.8,126.6,127.0,129.27(2 \mathrm{C})$, 129.28 (2C), 129.4 (2C), 129.7 (2C), 134.0, 139.2, 143.6, 146.4, 193.1; HRMS (FAB): $m / z$ calcd for $\mathrm{C}_{19} \mathrm{H}_{21} \mathrm{O}_{2}$, 281.1536; found, $281.1540\left([\mathrm{M}+\mathrm{H}]^{+}\right)$.

$\left(2 S^{*}, 4 S^{*}\right)-3,4-D i h y d r o-4-m e t h o x y-6-p h e n y l-2-(2-p h e n y l e t h y l)-2 H$-pyran (14).

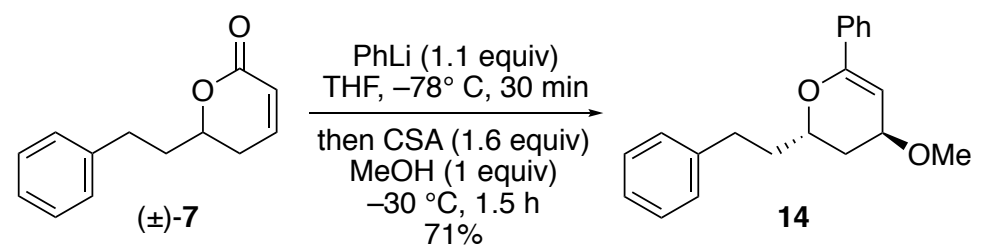

To a stirred solution of $( \pm)-7(56.3 \mathrm{mg}, 0.278 \mathrm{mmol})$ in THF $(2.8 \mathrm{~mL})$ was added dropwise a solution of PhLi $(1.6$ $\mathrm{M}$ in dibutyl ether, $0.19 \mathrm{~mL}, 0.306 \mathrm{mmol})$ at $-78{ }^{\circ} \mathrm{C}$. After $30 \mathrm{~min}$ of stirring, CSA (104 mg, $\left.0.448 \mathrm{mmol}\right)$ and $\mathrm{MeOH}(11.2 \mu \mathrm{L}, 0.278 \mathrm{mmol})$ were successively added to the mixture at $-78{ }^{\circ} \mathrm{C}$ and the cooling bath temperature was quickly raised from $-78{ }^{\circ} \mathrm{C}$ to $-30{ }^{\circ} \mathrm{C}$. After $1.5 \mathrm{~h}$ of stirring at $-30{ }^{\circ} \mathrm{C}$, the reaction mixture was quenched with satd aq $\mathrm{NaHCO}_{3}$ and extracted with EtOAc. The extract was washed with brine, dried $\left(\mathrm{MgSO}_{4}\right)$, and concentrated in vacuo. The residue $\left(\mathbf{1 4} / \mathbf{1 3}=\right.$ ca $6: 1$, as determined by ${ }^{1} \mathrm{H}$ NMR analysis) was purified by silica gel column chromatography (hexane/EtOAc $=15: 1)$ to give $14(57.7 \mathrm{mg}, 71 \%)$ as a colorless oil. IR: $v_{\max } 3060(\mathrm{w})$, 3026 (w), 1644 (m), 1495 (w), 1281 (m), 1083 (s); ${ }^{1} \mathrm{H}$ NMR (400 MHz, $\left.\mathrm{C}_{6} \mathrm{D}_{6}\right): \delta 1.39$ (1H, ddd, $J=4.2,12.2$, $14.1 \mathrm{~Hz}), 1.71(1 \mathrm{H}$, dddd, $J=4.2,6.3,10.2,13.8 \mathrm{~Hz}), 1.82(1 \mathrm{H}$, dddd, $J=1.6,1.8,1.8,14.1 \mathrm{~Hz}), 1.95(1 \mathrm{H}, \mathrm{dddd}$, $J=5.4,8.7,10.2,13.8 \mathrm{~Hz}), 2.67(1 \mathrm{H}, \mathrm{ddd}, J=6.3,10.2,13.8 \mathrm{~Hz}), 2.88(1 \mathrm{H}, \mathrm{ddd}, J=5.4,10.2,13.8 \mathrm{~Hz}), 3.16$ $(3 \mathrm{H}, \mathrm{s}), 3.61(1 \mathrm{H}, \mathrm{ddd}, J=1.8,4.2,5.4 \mathrm{~Hz}), 4.21(1 \mathrm{H}, \mathrm{dddd}, J=1.8,4.2,8.7,12.2 \mathrm{~Hz}), 5.60(1 \mathrm{H}, \mathrm{dd}, J=1.6,5.4$ $\mathrm{Hz}), 7.03-7.21(8 \mathrm{H}, \mathrm{m}), 7.72-7.76(2 \mathrm{H}, \mathrm{m}) ;{ }^{13} \mathrm{C} \mathrm{NMR}\left[100 \mathrm{MHz},\left(\mathrm{CD}_{3}\right)_{2} \mathrm{CO},\left(\mathrm{CD}_{3}\right)_{2} \mathrm{CO}\right.$ as an internal standard $(\delta$ $206.26 \mathrm{ppm})$ ]: $\delta 32.4,34.5,37.9,55.3,70.4,72.4,97.5,126.0(2 \mathrm{C}), 126.7,129.2(2 \mathrm{C}), 129.3(2 \mathrm{C}), 129.4(2 \mathrm{C})$, 129.5, 136.6, 143.0, 154.7; HRMS (EI): $m / z$ calcd for $\mathrm{C}_{20} \mathrm{H}_{22} \mathrm{O}_{2}, 294.1614$; found, $294.1622\left(\mathrm{M}^{+}\right)$. 
Preparation of Compounds A, B, and 7.

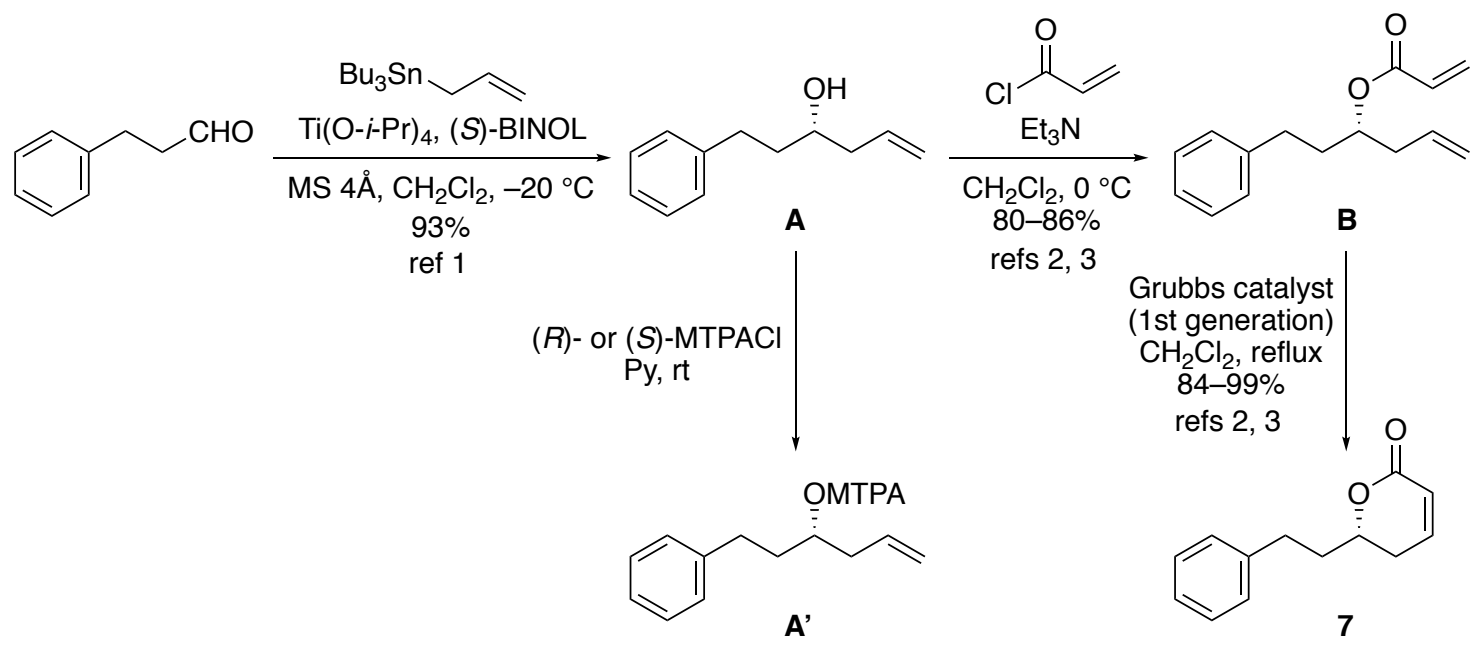

Compounds A, B, and 7 were prepared according to the literature procedures. ${ }^{1,2,3}$

1) Keck, G. E.; Krishnamurthy, D. Org. Synth. 1998, 75, 12-18.

2) Reddy, M. V. R.; Brown, H. C.; Ramachandran, P. V. J. Organomet. Chem. 2001, 624, 239-243.

3) de Fátima, A.; Kohn, L. K.; de Carvalho, J. E.; Pilli, R. A. Bioorg. Med. Chem. 2006, 14, 622-631.

(S)-1-Phenylhex-5-en-3-ol (A).

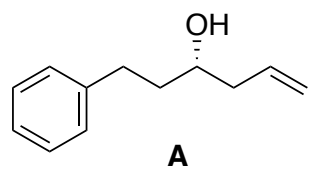

$[\alpha]^{20}{ }_{\mathrm{D}}-20.7\left(\mathrm{c} 2.26, \mathrm{CHCl}_{3}\right)$; IR: $v_{\max } 3381(\mathrm{~s}), 3077(\mathrm{w}), 3027(\mathrm{~m}), 1640(\mathrm{w}), 1496(\mathrm{w})$; ${ }^{1} \mathrm{H}$ NMR (400 MHz, $\left.\mathrm{CDCl}_{3}\right): \delta 1.65(1 \mathrm{H}, \mathrm{br} \mathrm{s}), 1.75-1.83(2 \mathrm{H}, \mathrm{m}), 2.14-2.23(1 \mathrm{H}, \mathrm{m})$, 2.29-2.37 (1H, m), 2.69 (1H, ddd, $J=7.4,9.0,13.6 \mathrm{~Hz}), 2.81(1 \mathrm{H}$, ddd, $J=6.8,8.8,13.6$ $\mathrm{Hz}), 3.64-3.71(1 \mathrm{H}, \mathrm{m}), 5.11-5.18(2 \mathrm{H}, \mathrm{m}), 5.76-5.88(1 \mathrm{H}, \mathrm{m}), 7.16-7.23(3 \mathrm{H}, \mathrm{m}), 7.25-7.31(2 \mathrm{H}, \mathrm{m}) ;{ }^{13} \mathrm{C} \mathrm{NMR}$ (100 MHz, $\left.\mathrm{CDCl}_{3}\right): \delta 32.1,38.5,42.2,70.0,118.4,125.9,128.50$ (2C), 128.54 (2C), 134.7, 142.2; HRMS (EI): $m / z$ calcd for $\mathrm{C}_{12} \mathrm{H}_{16} \mathrm{O}, 176.1196$; found, $176.1203\left(\mathrm{M}^{+}\right)$.

(S)-1-Phenylhex-5-en-3-yl acrylate (B).

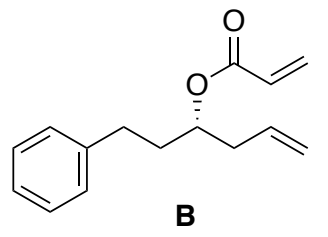

$[\alpha]^{20}{ }_{D}-11.0\left(c 2.47, \mathrm{CHCl}_{3}\right)$; IR: $v_{\max } 3080(\mathrm{w}), 3028(\mathrm{w}), 1723(\mathrm{vs}), 1406(\mathrm{~m}), 1197(\mathrm{~s}) ;$ ${ }^{1} \mathrm{H}$ NMR (400 MHz, $\left.\mathrm{CDCl}_{3}\right): \delta 1.85-2.00(2 \mathrm{H}, \mathrm{m}), 2.36-2.42(2 \mathrm{H}, \mathrm{m}), 2.61(1 \mathrm{H}, \mathrm{ddd}, J=$ 7.0, 9.6, $14.2 \mathrm{~Hz}), 2.69(1 \mathrm{H}, \mathrm{ddd}, J=6.4,9.8,14.2 \mathrm{~Hz}), 5.01-5.12(3 \mathrm{H}, \mathrm{m}), 5.76(1 \mathrm{H}, \mathrm{ddt}$, $J=10.2,17.2,7.1 \mathrm{~Hz}), 5.83(1 \mathrm{H}, \mathrm{dd}, J=1.6,10.5 \mathrm{~Hz}), 6.12(1 \mathrm{H}, \mathrm{dd}, J=10.5,17.3 \mathrm{~Hz})$, $6.41(1 \mathrm{H}, \mathrm{dd}, J=1.6,17.3 \mathrm{~Hz}), 7.14-7.21(3 \mathrm{H}, \mathrm{m}), 7.26-7.30(2 \mathrm{H}, \mathrm{m}) ;{ }^{13} \mathrm{C} \mathrm{NMR}\left(100 \mathrm{MHz}, \mathrm{CDCl}_{3}\right): \delta 31.8,35.5$, 38.8, 73.2, 118.1, 126.1, 128.5 (2C), 128.6 (2C), 128.9, 130.7, 133.5, 141.6, 166.0; HRMS (FAB): $\mathrm{m} / z$ calcd for $\mathrm{C}_{15} \mathrm{H}_{19} \mathrm{O}_{2}, 231.1380$; found, $231.1381\left([\mathrm{M}+\mathrm{H}]^{+}\right)$.

(S)- 5,6-Dihydro-6-(2-phenylethyl)-2H-pyran-2-one (7).

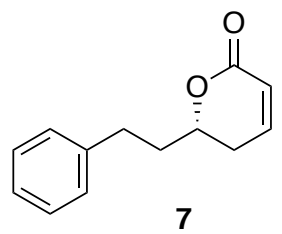

$[\alpha]_{\mathrm{D}}^{20}+45.1\left(\mathrm{c} 1.27, \mathrm{CHCl}_{3}\right)$; IR: $v_{\max } 3060(\mathrm{w}), 3027(\mathrm{w}), 1716(\mathrm{~s}), 1246(\mathrm{~m}), 1037(\mathrm{w}) ;{ }^{1} \mathrm{H}$ NMR (400 MHz, $\left.\mathrm{CDCl}_{3}\right): \delta 1.89-1.99(1 \mathrm{H}, \mathrm{m}), 2.09-2.20(1 \mathrm{H}, \mathrm{m}), 2.28-2.42(2 \mathrm{H}, \mathrm{m}), 2.78$ (1H, ddd, $J=7.3,9.1,14.2 \mathrm{~Hz}), 2.90(1 \mathrm{H}, \mathrm{ddd}, J=5.3,9.1,14.2 \mathrm{~Hz}), 4.37-4.46(1 \mathrm{H}, \mathrm{m})$, $6.03(1 \mathrm{H}, \mathrm{ddd}, J=1.6,2.2,10.0 \mathrm{~Hz}), 6.84-6.90(1 \mathrm{H}, \mathrm{m}), 7.18-7.24(3 \mathrm{H}, \mathrm{m}), 7.27-7.33(2 \mathrm{H}$, $\mathrm{m}) ;{ }^{13} \mathrm{C}$ NMR (100 MHz, $\mathrm{CDCl}_{3}$ ): $\delta$ 29.6, 31.1, 36.7, 77.0, 121.6, 126.3, 128.6 (2C), 128.7 (2C), 141.0, 145.1, 164.6; HRMS (FAB): $m / z$ calcd for $\mathrm{C}_{13} \mathrm{H}_{15} \mathrm{O}_{2}, 203.1067$; found, $203.1069\left([\mathrm{M}+\mathrm{H}]^{+}\right)$. 


\section{Determination of the enantiomeric excess of $A$.}

Compound A was converted into the corresponding (R)- and $(S)$ - MTPA esters by treatment with (S)- and $(R)$-MTPA chloride, respectively, in pyridine. By comparing the peak areas of the ${ }^{1} \mathrm{H}$ NMR signals for OCH $\boldsymbol{H}_{3}$ (3.42 and $3.46 \mathrm{ppm}$ ) of the esters, the enantiomeric excess of $\mathbf{A}$ was evaluated to be $96 \%$ (see S10).

\section{Diocollettines A (1).}

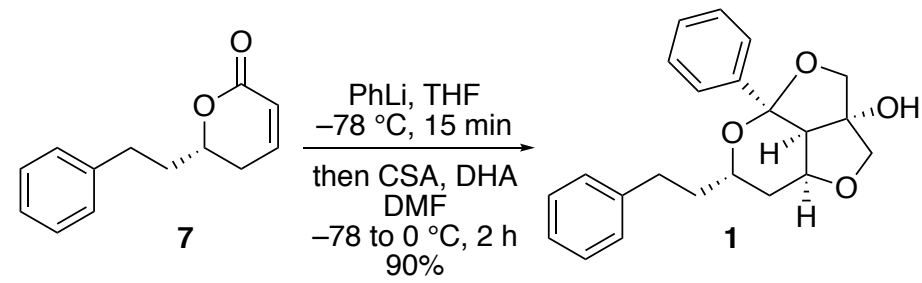

To a stirred solution of $7(27.3 \mathrm{mg}, 0.135 \mathrm{mmol})$ in THF $(1.4 \mathrm{~mL})$ was added dropwise a solution of PhLi (1.6 M in dibutyl ether, $84 \mu \mathrm{L}, 0.135 \mathrm{mmol}$ ) at $-78{ }^{\circ} \mathrm{C}$. After $15 \mathrm{~min}$ of stirring, CSA (94.4 $\mathrm{mg}, 0.406 \mathrm{mmol}$ ), 1,3-dihydroxyacetone dimer $(61.2 \mathrm{mg}, 0.340 \mathrm{mmol})$ and distilled DMF $(0.5 \mathrm{~mL})$ were successively added at $-78{ }^{\circ} \mathrm{C}$ and then the cooling bath was quickly changed to an ice-water bath. After $2 \mathrm{~h}$ of stirring, the reaction mixture was quenched with satd aq $\mathrm{NaHCO}_{3}$ and extracted with EtOAc. The extract was successively washed with water and brine, dried $\left(\mathrm{MgSO}_{4}\right)$, and concentrated in vacuo. The residue was purified by silica gel column chromatography (hexane/EtOAc $=7: 3)$ to give $1(42.9 \mathrm{mg}, 90 \%)$ as a white solid. Mp $127.3-128.8{ }^{\circ} \mathrm{C} ;[\alpha]^{20}{ }_{\mathrm{D}}$ -125.1 (c 0.185, toluene); IR: $v_{\max } 3434$ (m), 3060 (w), 3027 (w), 1603 (w), 1064 (s); ${ }^{1} \mathrm{H}$ NMR [600 MHz, $\left(\mathrm{CD}_{3}\right)_{2} \mathrm{SO}, \mathrm{CD}_{3} \mathrm{~S}(\mathrm{O}) \mathrm{CD}_{2} H$ as an internal standard $\left.(\delta 2.50 \mathrm{ppm})\right]: \delta 1.61-1.75(3 \mathrm{H}, \mathrm{m}), 1.81(1 \mathrm{H}, \mathrm{d}, J=14.4 \mathrm{~Hz})$, 2.59-2.67 (3H, m), $3.51(1 \mathrm{H}, \mathrm{d}, J=8.4 \mathrm{~Hz}), 3.77(1 \mathrm{H}, \mathrm{d}, J=9.2 \mathrm{~Hz}), 3.91(1 \mathrm{H}, \mathrm{d}, J=8.4 \mathrm{~Hz}), 3.96-4.00(1 \mathrm{H}, \mathrm{m})$, $4.02(1 \mathrm{H}, \mathrm{d}, J=9.0 \mathrm{~Hz}), 4.29-4.31(1 \mathrm{H}, \mathrm{m}), 5.48(1 \mathrm{H}, \mathrm{s}), 7.14-7.32(6 \mathrm{H}, \mathrm{m}), 7.37(2 \mathrm{H}, \mathrm{t}, J=7.8 \mathrm{~Hz}), 7.55(2 \mathrm{H}, \mathrm{d}$, $J=7.8 \mathrm{~Hz}) ;{ }^{13} \mathrm{C}$ NMR $\left[150 \mathrm{MHz},\left(\mathrm{CD}_{3}\right)_{2} \mathrm{SO},\left(\mathrm{CD}_{3}\right)_{2} \mathrm{SO}\right.$ as an internal standard $\left.(\delta 39.52 \mathrm{ppm})\right]: \delta 30.9,32.1,36.8$, 52.6, 63.7, 75.1, 76.7, 78.3, 87.7, 107.5, 125.7, 125.8 (2C), 127.6, 127.9 (2C), 128.3 (2C), 128.4 (2C), 141.9, 144.6; HRMS (FAB): $m / z$ calcd for $\mathrm{C}_{22} \mathrm{H}_{25} \mathrm{O}_{4}, 353.1747$; found, $353.1758\left([\mathrm{M}+\mathrm{H}]^{+}\right)$. 
NMR spectra
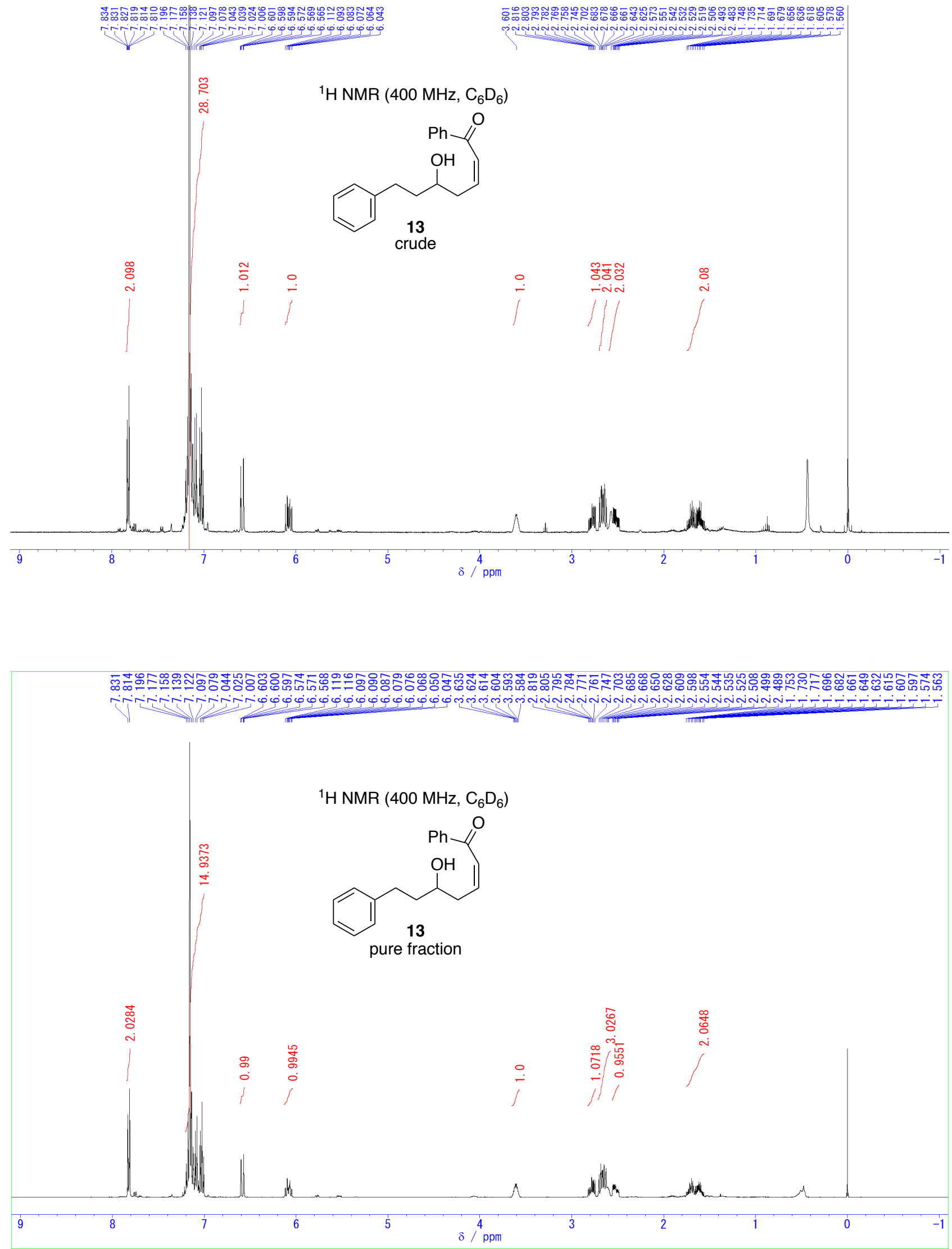

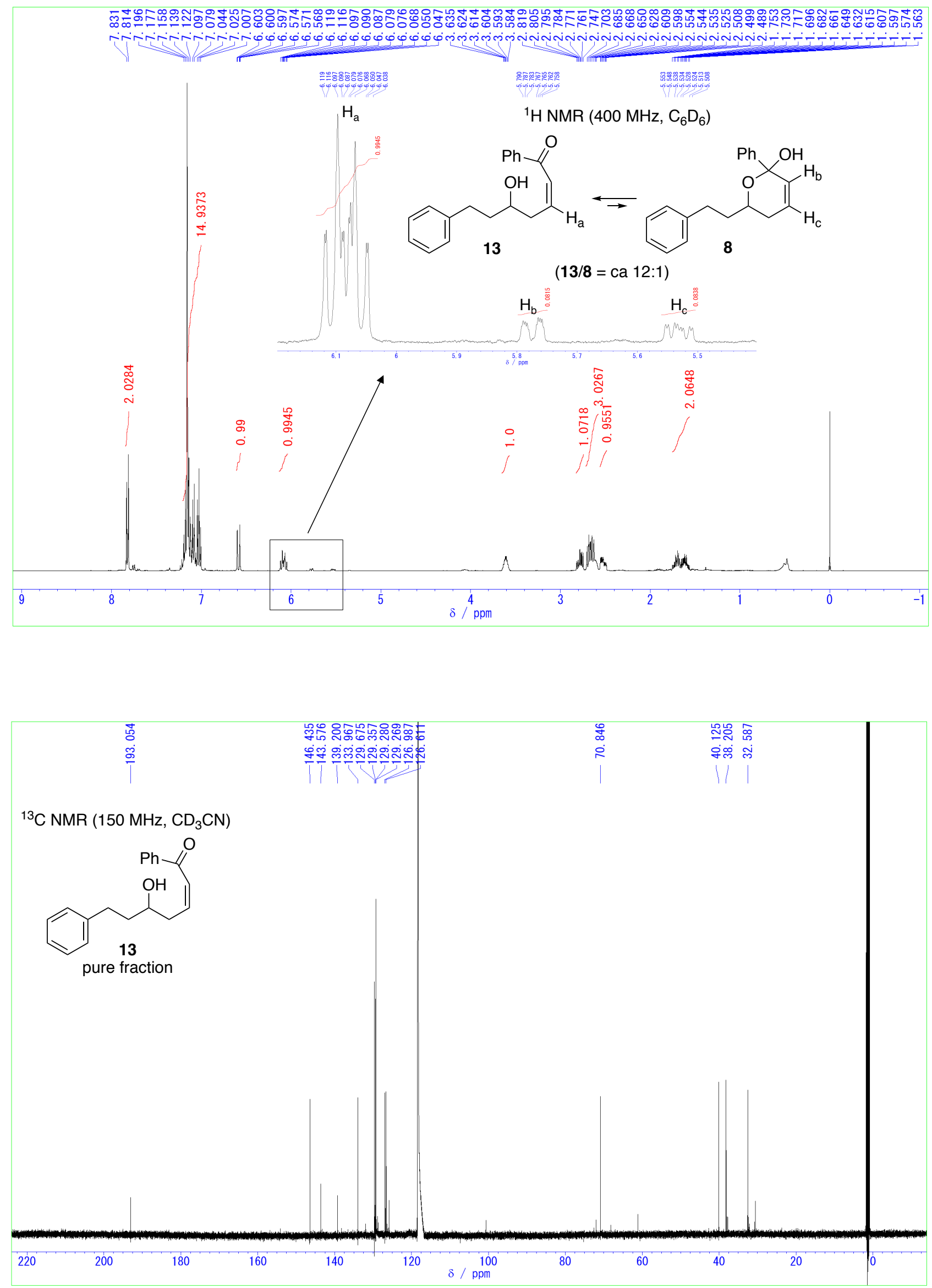

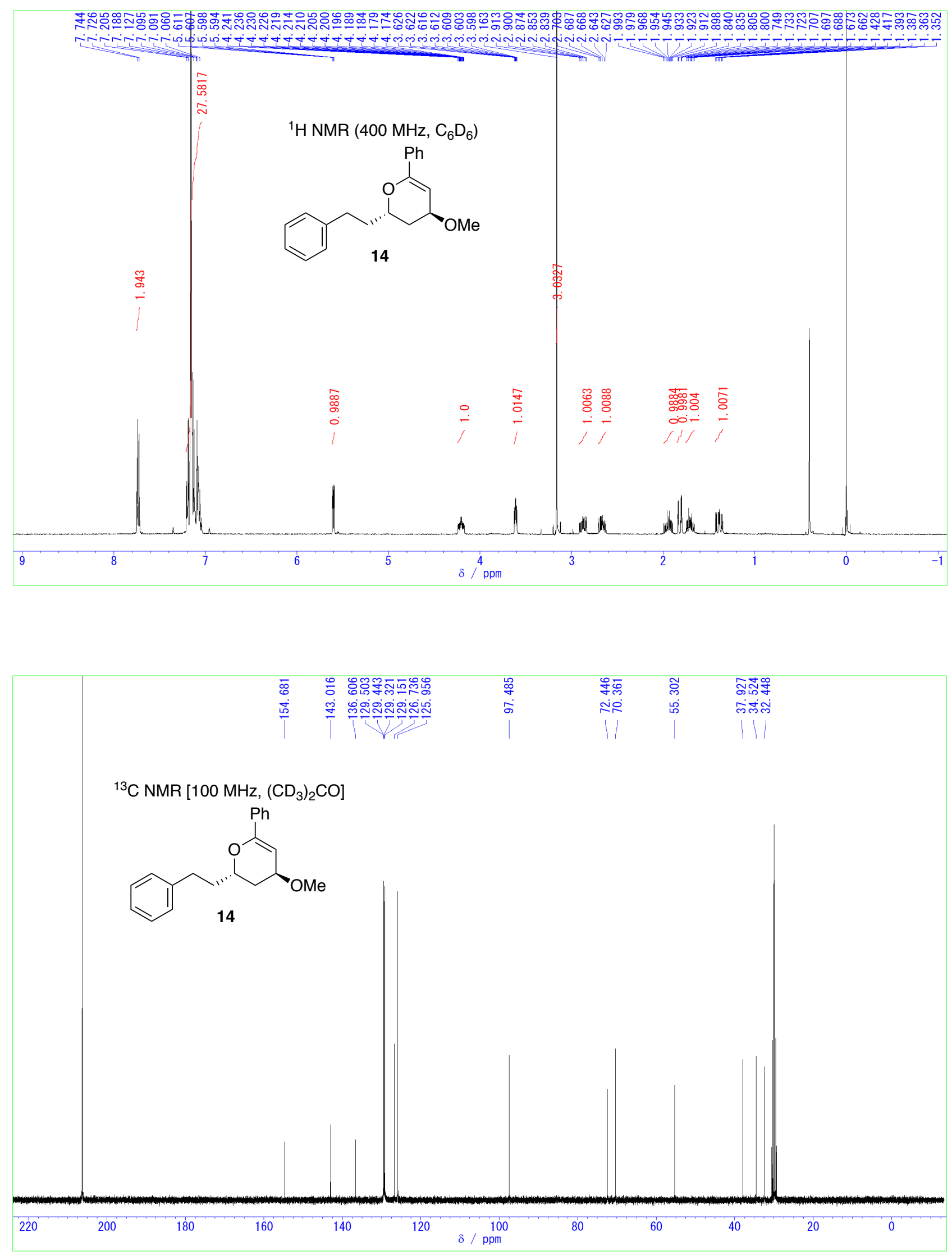

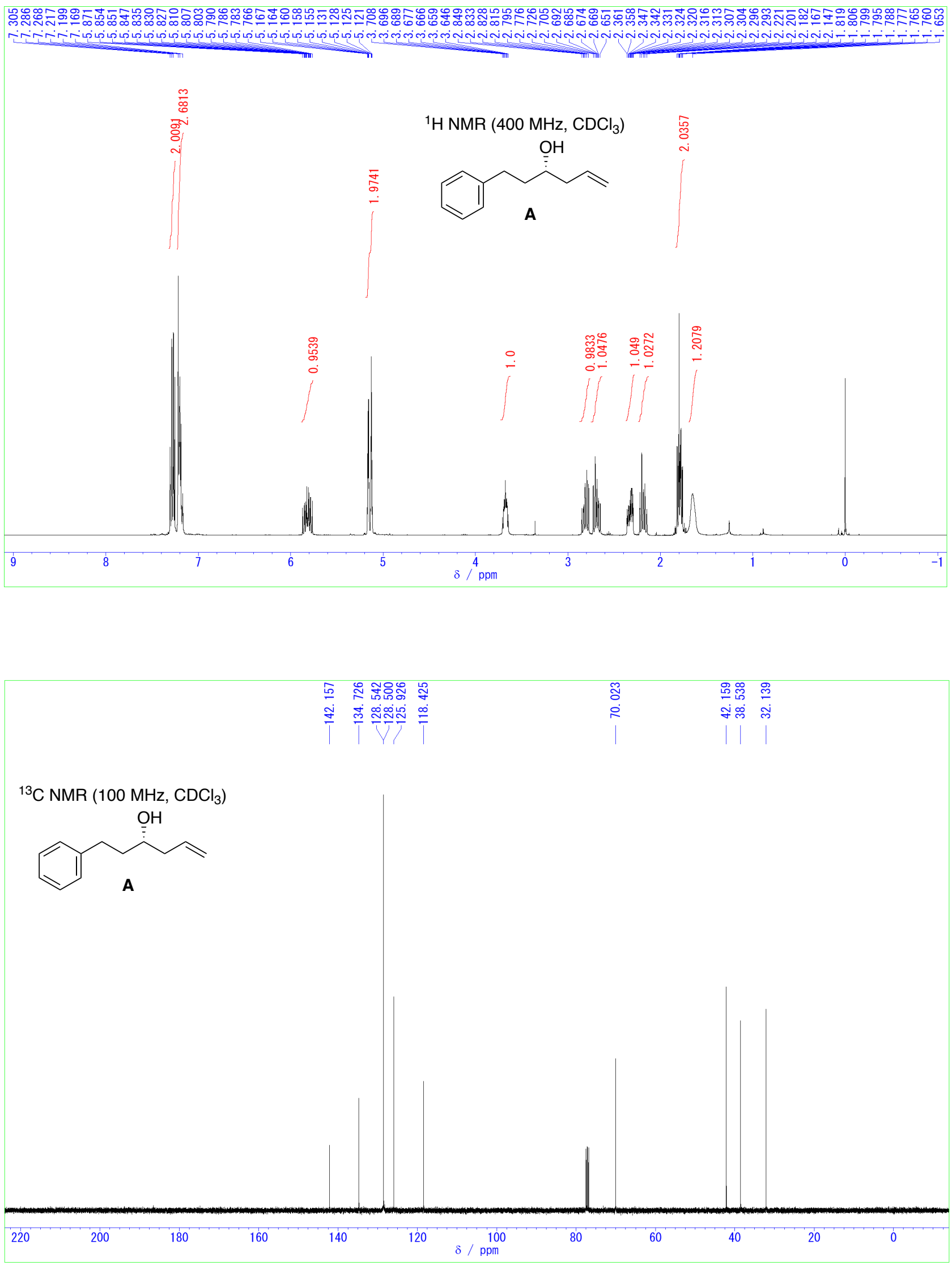

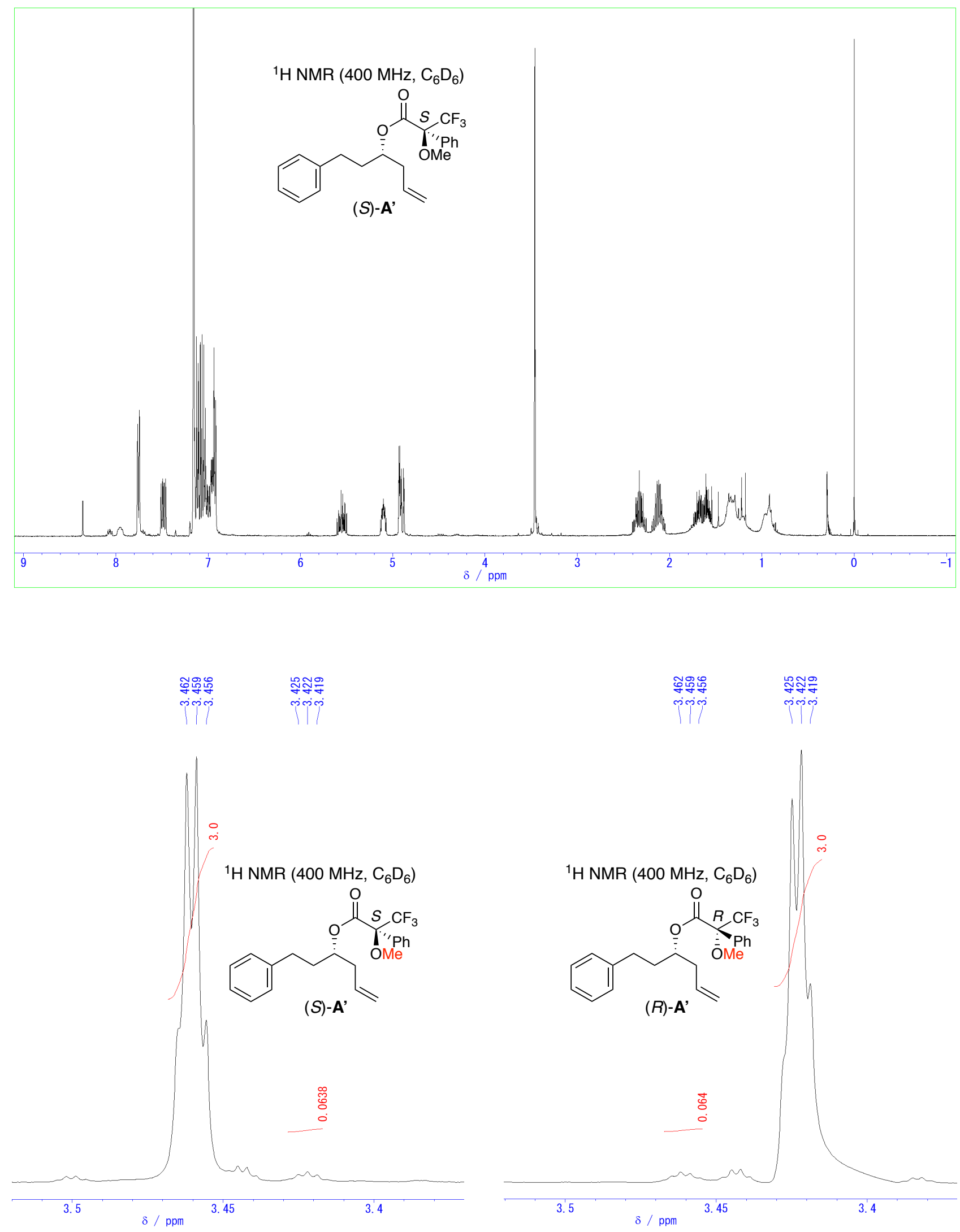

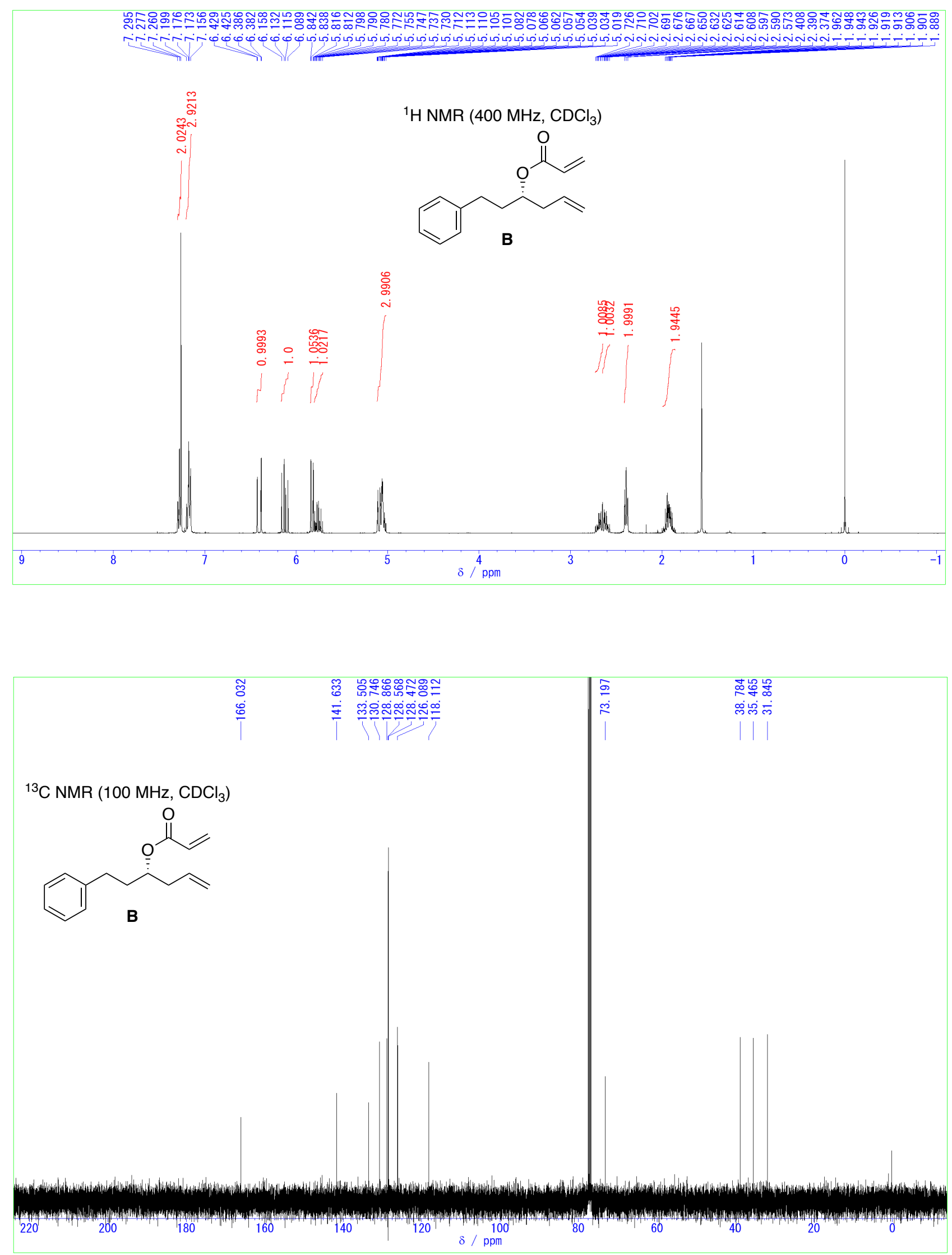

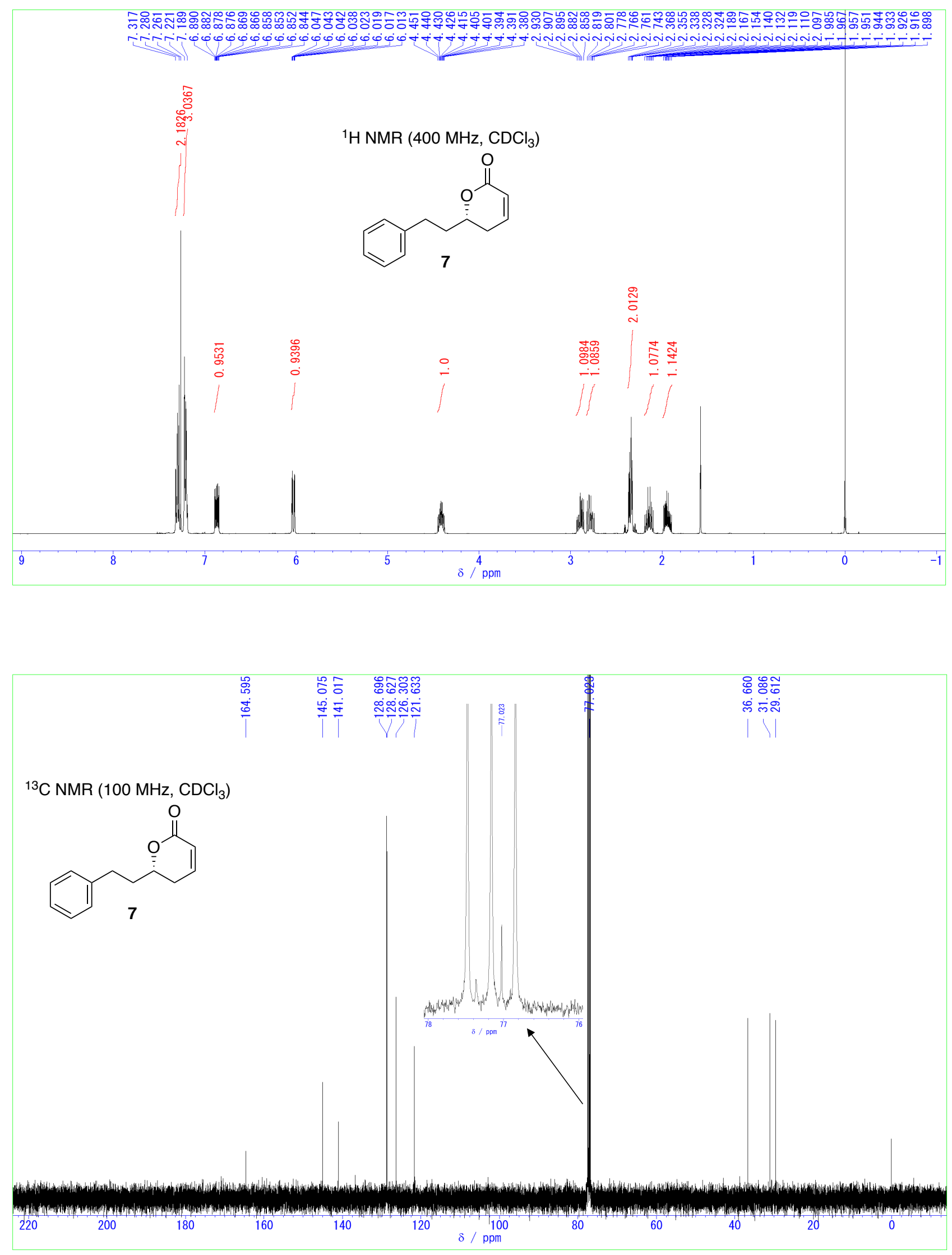

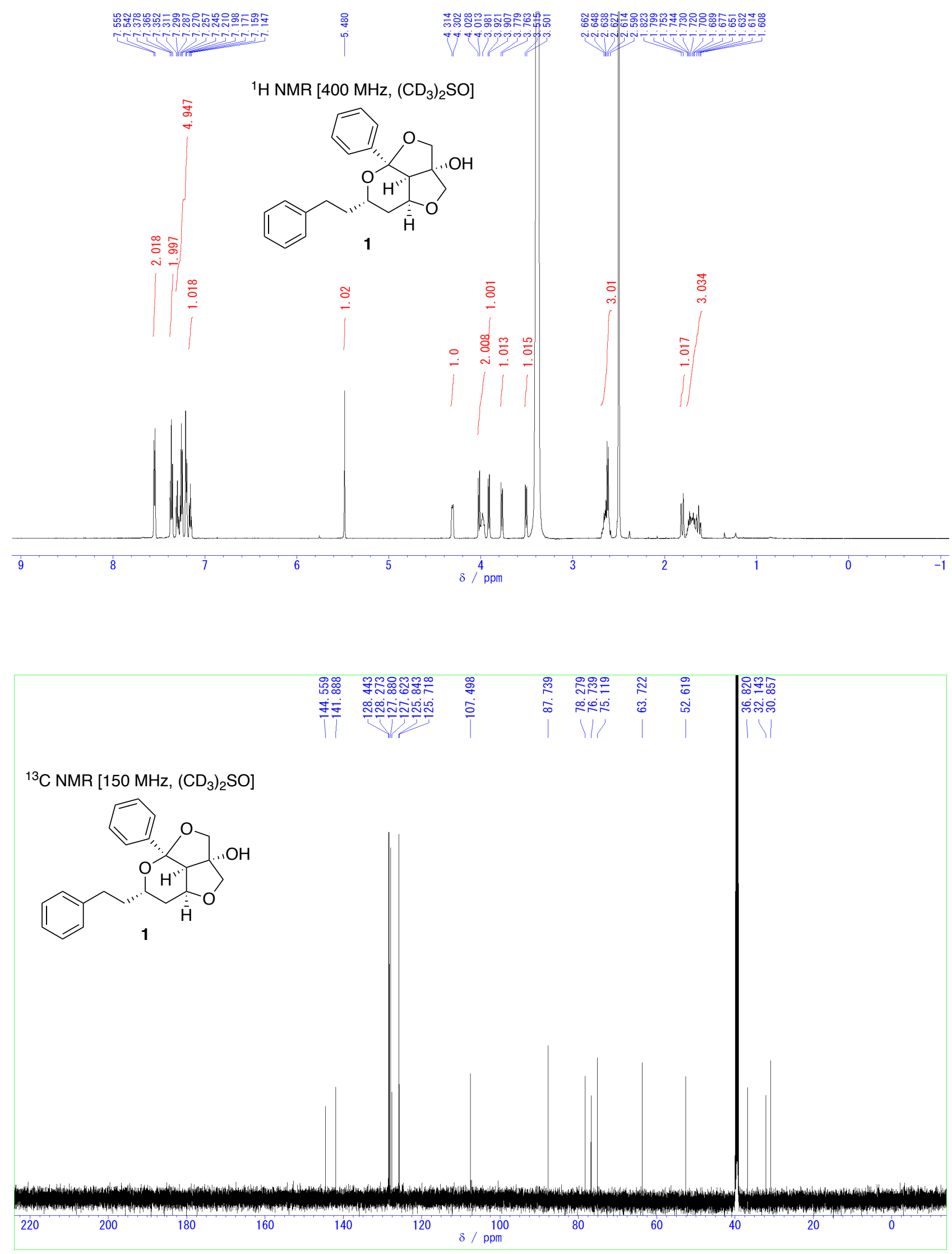